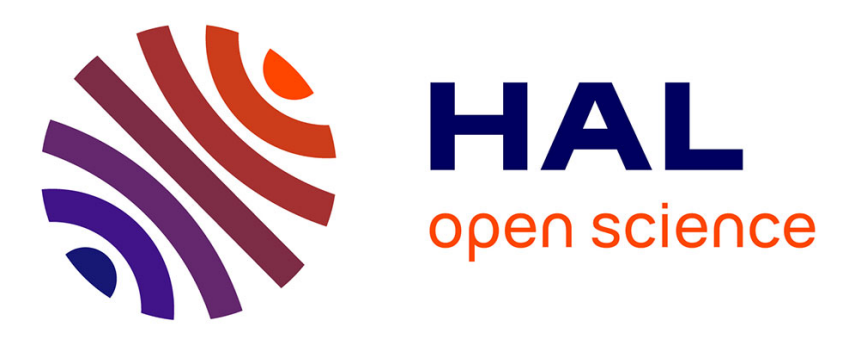

\title{
Influence of the Reverberation Chamber on Antenna Characterization Performances
}

\author{
W. Krouka, François Sarrazin, Elodie Richalot
}

\section{To cite this version:}

W. Krouka, François Sarrazin, Elodie Richalot. Influence of the Reverberation Chamber on Antenna Characterization Performances. 2018 International Symposium on Electromagnetic Compatibility (EMC EUROPE), Aug 2018, Amsterdam, Netherlands. 10.1109/EMCEurope.2018.8485064 . hal01933289

\section{HAL Id: hal-01933289 \\ https://hal.science/hal-01933289}

Submitted on 23 Nov 2018

HAL is a multi-disciplinary open access archive for the deposit and dissemination of scientific research documents, whether they are published or not. The documents may come from teaching and research institutions in France or abroad, or from public or private research centers.
L'archive ouverte pluridisciplinaire HAL, est destinée au dépôt et à la diffusion de documents scientifiques de niveau recherche, publiés ou non, émanant des établissements d'enseignement et de recherche français ou étrangers, des laboratoires publics ou privés. 


\title{
Influence of the reverberation chamber on antenna characterization performances
}

\author{
W. Krouka, F. Sarrazin, E. Richalot \\ Université Paris-Est, ESYCOM (EA \\ 2552), UPEMLV, ESIEE-Paris, CNAM, \\ F-77454 Marne-la-Vallée, France \\ wafa.krouka@u-pem.fr
}

\begin{abstract}
Antenna characterization using reverberation chamber is being widely used and therefore several measurement techniques have been proposed. In this paper, the characterization technique based on two antennas is used in order to determine the radiation efficiency of a patch antenna. This work is conducted in two different configurations of a reverberation chamber (classical configuration and chaotic configuration). We evaluate the stirring techniques used (mechanical and frequential) in both configurations by calculating the number of independent samples. Statistical behavior of both cavity configurations (field homogeneity and isotropy) are also evaluated through the calculation of the enhanced backscatter parameter and the standard deviation of the measured parameters. Different measurements and calculation results are compared.
\end{abstract}

Keywords-Antenna characterization, reverberation chamber, mode stirring, chaoticity.

\section{INTRODUCTION}

Reverberation Chamber (RC) measurements have been extended to antenna characterization after being mainly limited to electromagnetic compatibility measurements. Indeed, $\mathrm{RC}$ is an interesting alternative to Anechoic Chamber (AC) to perform antenna radiation efficiency measurement. The original approach consists in measuring the power received by an antenna under test and comparing it to the power received by a reference antenna of known efficiency [1]. Other techniques, based on quality factor measurement, avoid the use of a reference antenna [2] [3]. RC measurement accuracy lays on the uniform and isotropic incident field impinging on the antenna under test within the usable volume. These ideal properties are only obtained statistically by averaging the field over a number of independent realizations.

There are two main mode stirring techniques that allow obtaining different field configurations inside the RC. Mechanical stirring permits to change the boundary conditions of the electromagnetic field inside the $\mathrm{RC}$ using a rotating metallic stirrer. Besides, frequency stirring is also used by taking measurement samples over a frequency range. Stirring techniques and even their combination have been studied in [4] [5]. The random behavior of the electrical field inside the $\mathrm{RC}$ in addition to the number of measurements obtained through stirring allows us to apply statistical treatment on the electrical field.

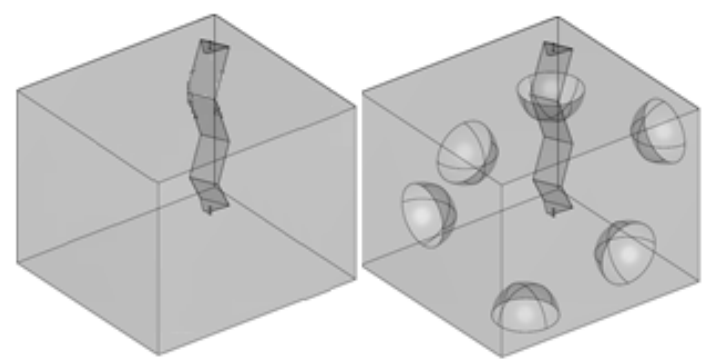

(a)

(b)

Fig. 1. Reverberation chamber configurations: (a) classical configuration (b) chaotic configuration.

Recently, chaotic cavities have aroused great interest in the $\mathrm{RC}$ community [6] as they present intrinsically uniform and isotropic field (without the need of any stirring process) compared to classical parallelepiped regular cavities used as RC. The objective of this paper is to evaluate the accuracy improvement of antenna efficiency measurement performed inside a chaotic RC compared to a classical one.

Measurements presented in this paper are conducted in two different configurations of an RC (2.95 $\mathrm{m} \times 2.95 \mathrm{~m} \times 2.35 \mathrm{~m})$ : the classical configuration (Fig. 1.a) and the chaotic configuration (Fig. 1.b) in which the geometry of the walls inside the $\mathrm{RC}$ has been modified by adding six metallic hemispheres (of radius $40 \mathrm{~cm}$ ) in order to make its behavior more chaotic. Previous studies have already been conducted in this context [6].

\section{ANTENNA EFFICIENCY MEASUREMENT TECHNIQUE}

In [2] different RC measurement techniques for determining antenna efficiency have been exposed. The approach based on two antennas is applied throughout this paper as it presents a good tradeoff between measurement accuracy and complexity. Two identical patch antennas are considered. They are made of a half-disc slot excited by a monopole and printed on FR4 substrate (Fig. 2). They are broadband antennas matched between 1.75 and $2.7 \mathrm{GHz}\left(\mathrm{S}_{11}\right.$ minor than $\left.-6 \mathrm{~dB}\right)$, so their operating frequencies are much higher than the Lowest Usable Frequency (LUF) of the RC (around $400 \mathrm{MHz}$ ). 

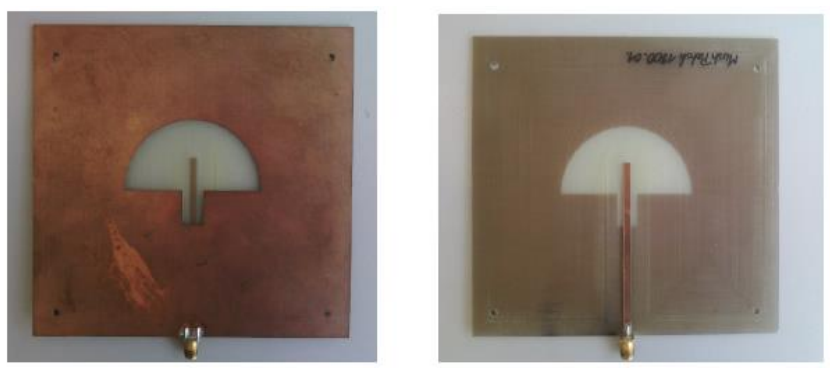

Fig. 2. Characterized patch antenna.

In order to determine the antenna efficiency, we need to calculate the chamber's time constant $\tau_{\mathrm{RC}}$ and the backscatter coefficient $e_{b}$. The parameter $\tau_{\mathrm{RC}}$ represents the delay time that takes the energy inside the chamber to reverberate. It is determined from the power delay profile $(P D P)$ as the inverse of its slope. The latter is calculated using the chamber's time domain response generally obtained using scattering parameter $\mathrm{S}_{21}(1)$.

$$
\begin{gathered}
P D P(t)=\left\langle|h(t, n)|^{2}\right\rangle \\
h(t, n)=\operatorname{IFT}\left[S_{21_{n}}(f)\right]
\end{gathered}
$$

with ' $n$ ' the number of independent samples obtained using stirring techniques.

$\mathrm{RC}$ quality factor is mainly related to different losses in the chamber. It can be calculated from the chamber's time constant $\tau_{\mathrm{RC}}(3)$.

$$
Q=\omega \tau_{R C}
$$

with $\omega=2 \pi f$ ( $f$ is the frequency). As electric field in $\mathrm{RC}$, measured scattering parameters within an RC can be decomposed into stirred and unstirred components.

$$
S_{i j}=S_{i j, s}+S_{i j, u s}
$$

with $i, j=\{1,2\}$, the indexes relative to the first and the second antenna. The unstirred component represents the part that is not influenced by the stirrer position and is deterministic (ideally equal to the measured parameter in $\mathrm{AC}$ ). On the other hand, stirred component is the statistical random part. It is calculated using (5). In our case, uncorrelated component is obtained by calculating the average of the measured parameter in $\mathrm{RC}$ over all stirrer positions.

$$
S_{i j, s}=S_{i j}-\left\langle S_{i j}\right\rangle
$$

Using stirred components of the measured scattering parameters, we can calculate the enhanced backscatter coefficient (6). This coefficient evaluates the homogeneity of the electrical field inside the $\mathrm{RC}$ and is theoretically equal to 2 if the used reverberation chamber is well stirred [7]. In our case, the used antennas are considered identical. Thus, we consider
$\left\langle\left|S_{22, s}\right|^{2}\right\rangle$ and $\left\langle\left|S_{11, s}\right|^{2}\right\rangle$ as equivalent, and the enhanced backscattering coefficient is then calculated as (6).

$$
e_{b}=\frac{\left\langle\left|S_{11, s}\right|^{2}\right\rangle}{\left\langle\left|S_{21, s}\right|^{2}\right\rangle}
$$

After calculating the previous quantities, antenna efficiency can be determined using (7) for the total antenna efficiency.

$$
\eta_{t o t}=\sqrt{\frac{C_{R C}}{\omega e_{b}} \frac{\left\langle\left|S_{11, s}\right|^{2}\right\rangle}{\tau_{R C}}}
$$

where $C_{R C}$ is a frequency dependent chamber's constant and is equal to $\frac{16 \pi^{2} V}{\lambda^{3}}$, ' $V$ ' being the RC volume and ' $\lambda$ ' the wavelength. In order to obtain antenna radiation efficiency, we apply a correction to the scattering parameter dependent quantity $\left\langle\left|S_{11, s}\right|^{2}\right\rangle$. This correction aims to overcome the mismatch of the antenna.

\section{STIRRING PERFORMANCES}

In order to evaluate the measurement accuracy in an $\mathrm{RC}$, the patch antenna under test is first of all characterized in an $\mathrm{AC}$; the obtained reflection coefficient will serve as a reference in the following. Measurements are then performed in the RC using a Rohde\&Schwarz ZNB8 VNA over the $1 \mathrm{GHz}-3.5 \mathrm{GHz}$ frequency band.

Mechanical and frequency stirring techniques are applied to the measured parameters in $\mathrm{RC}$ in order to enhance measurements precision. To better understand the impact of both stirring techniques, they are compared in both configurations of the $\mathrm{RC}$ in the next two parts.

\section{A. Mechanical Stirring}

Mechanical stirring consists in rotating the stirrer and performing measurements for each position of the stirrer. In our case, we use a rotation step of 5 degrees; thus, a complete rotation of the stirrer provides us with 72 measurements.

In order to calculate the number of uncorrelated stirrer positions we evaluated the correlation coefficient between the received power at a fixed antenna location versus the stirrer rotation angle. Adopting the same criterion as in [3] and [4], we consider that data are uncorrelated once the correlation coefficient is lower than $1 / e$. The Fig. 3 presents the correlation coefficient calculated over a $600 \mathrm{MHz}$ window centered on $2 \mathrm{GHz}$, the number of independent stirrer positions is estimated to be 72 in both cavity configurations, i.e. all positions that have been actually measured can be considered as uncorrelated. 


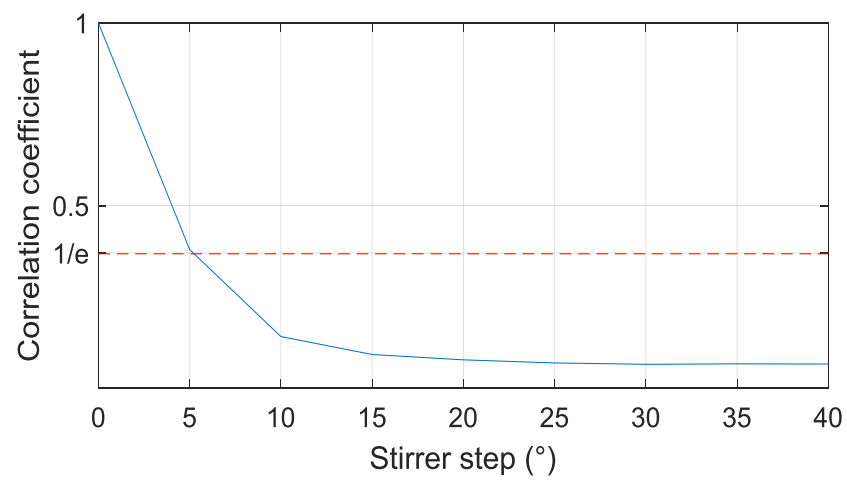

Fig. 3. Correlation coefficient versus stirrer step between received power for a fixed antenna location in chaotic RC over a $600 \mathrm{MHz}$ window centered on $2 \mathrm{GHz}$.

In order to study the impact of mechanical stirring on measurements in RC we calculate the STandard Deviation (STD) of the mechanical stirred $S_{11}$ relatively to the measurements of the same parameter in the AC (Tab. 1). We can notice the lower fluctuation level in the chaotic RC configuration compared to the classical RC.

TABLE I. STANDARD DEVIATION OF COMPLEX MECHANICAL STIRRED SCATTERING PARAMETER $S_{11}$ IN REGARD TO THE COEFFICIENT MEASURED IN AC.

\begin{tabular}{|l|c|c|}
\hline RC configuration & Classical & Chaotic \\
\hline SDT without stirring & 0.1101 & 0.09 \\
\hline SDT with stirring & 0.0853 & 0.0560 \\
\hline
\end{tabular}

Calculation results of STD present lower values for both chamber configurations when mechanical stirring is applied relatively to results calculated for one fixed stirrer position (without stirring). Also, we clearly notice that the chaotic configuration presents better performances even when mechanical stirring is not applied, which is consistent with the chaotic cavity theory. We note that the electrical field homogeneity can be enhanced simply by making the RC structure more chaotic.

\section{B. Frequency stirring}

To emulate the effect of a frequency stirring process, the measured complex reflection coefficient is averaged over a sliding window of a chosen width and the result is associated to the central frequency of the averaging bandwidth. As the frequency bandwidth is usually arbitrary chosen, we suggest to study its impact on the results in terms of STD compared to the $\mathrm{AC}$. It is equivalent to vary the number of uncorrelated data considered in the average calculation. Results are presented in Fig. 4. We observe that lower STD values are obtained in the chaotic RC configuration compared to the classical RC configuration, no matter the frequency stirrer window width. As expected, the STD decreases as we perform the frequency stirring for reasonable frequency widths. Then, it starts increasing because the considered window becomes very large compared to $S_{11}$ fluctuations. A minimal STD is obtained with a window of $106 \mathrm{MHz}$ in the classical configuration and $98 \mathrm{MHz}$ in the chaotic configuration (Fig. 4). Therefore an averaging window of $100 \mathrm{MHz}$ will be used in the following.

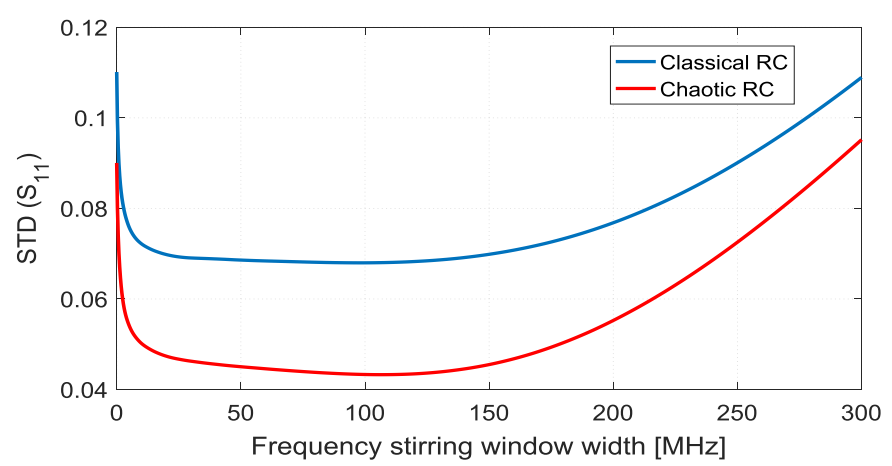

Fig. 4. Standard deviation of the complex $S_{11}$ parameter obtained in the RC and averaged over variable frequency windows relatively to $S_{11}$ coefficient measured in the anechoic chamber.

The Fig. 5 presents the frequency stirred antenna reflection coefficient obtained with a $100 \mathrm{MHz}$ bandwidth averaging window in both $\mathrm{RC}$ configurations and compares them to the reflection coefficient measured in an $\mathrm{AC}$. We notice that the curve obtained in the chaotic configuration is smoother than the one associated to the classical $\mathrm{RC}$, as expected from previous results.

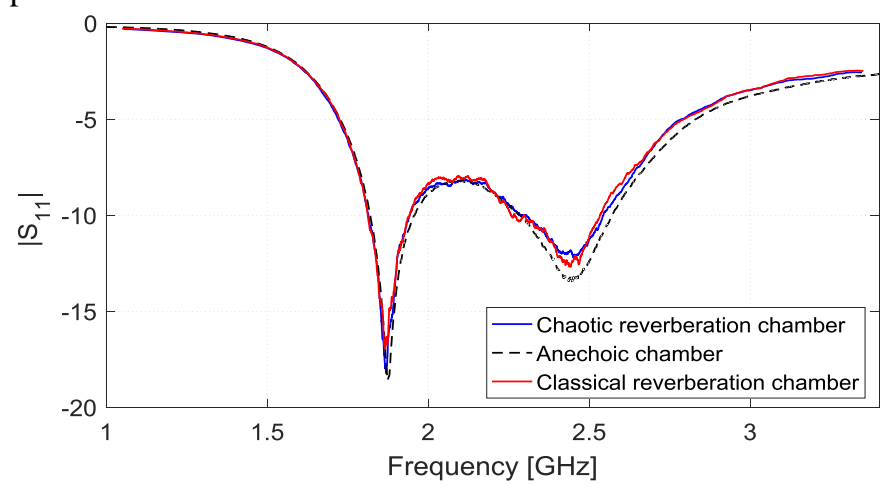

Fig. 5. Frequency stirred $S_{11}$ parameter using a $100 \mathrm{MHz}$ averaging bandwidth.

In order to evaluate the number of uncorrelated data over the averaging window we calculate the coherent bandwidth using the formula $\Delta f=f / Q$ [2] where ' $Q$ ' is the quality factor whose calculation will be detailed in Section (IV.A). Concerning the number of independent frequency samples, for a frequency of $2 \mathrm{GHz}$, the calculated number of uncorrelated samples over a $100 \mathrm{MHz}$ window is around 400 . This large value compared to the number of independent stirrer positions explains the better efficiency of the frequency stirring technique in both configurations. The enhancement of independent data samples number results in a better measurement precision.

This study on the frequency stirring shows the necessity to have a large number of uncorrelated samples to obtain a precise determination of the reflection coefficient. Therefore, in the following, to overcome the limitation of the low number of independent stirrer positions, the obtained parameters when considering the mechanical stirring are smoothed on a sliding frequency window of $100 \mathrm{MHz}$. Table 2 shows the good accuracy of the antenna reflection coefficient characterization using this approach. 
TABLE II. STANDARD DEVIATION OF MECHANICAL STIRRED AND SMOOTHED SCATTERING PARAMETER $\mathrm{S}_{11}$ USING $100 \mathrm{MHZ}$ SMOOTHING WINDOW WIDTH.

\begin{tabular}{|l|c|c|}
\hline RC configuration & Classical & Chaotic \\
\hline SDT without smoothing & 0.0853 & 0.0560 \\
\hline STD with smoothing & 0.0279 & 0.0258 \\
\hline
\end{tabular}

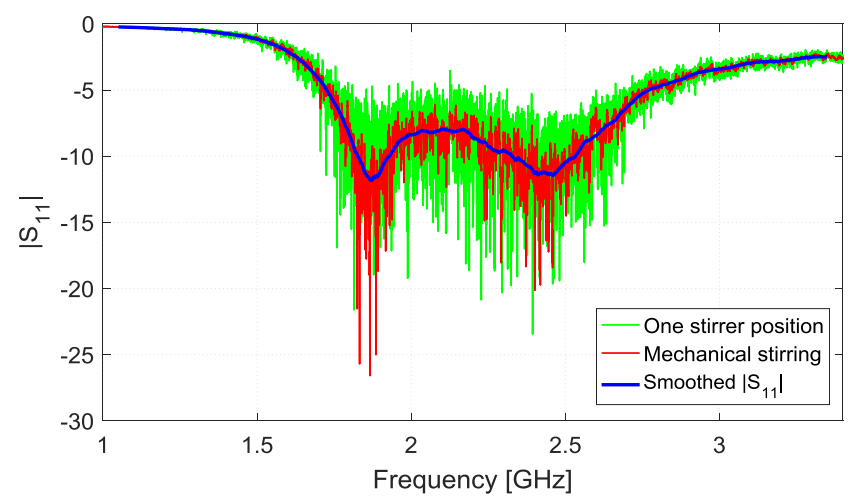

Fig. 6. Scattering parameter of the patch antenna in the chaotic RC without and after applying mechanical stirring techniques and frequency smoothing.

Large fluctuations are observed with measurements performed at a fixed stirrer position in the chaotic RC without considering any stirring technique (green curve). Mechanical stirring leads to a decrease of the response fluctuations (red curve). Moreover, a better result is obtained when applying, after the mechanical stirring step, a smoothing over a sliding window of $100 \mathrm{MHz}$ over all the frequency range (blue curve).

\section{ANTENNA EFFICIENCY CALCULATION}

\section{A. Quality factor}

Quality factor $Q$ has already been presented in Section II. Using (3), we calculate $Q$ in both RC configurations. Results are presented in Fig. 7. We notice a decrease of the quality factor in the chaotic RC configuration compared to the classical one. To understand this difference, we consider the quality factor associated to losses localized on the cavity walls in a well-operating $\mathrm{RC}$ (where the field is homogeneous). In this case, the quality factor can be approximated as

$$
Q=\frac{3}{2} \frac{V}{S \delta}
$$

where $\delta$ is the wall penetration depth. Thus, the quality factor is proportional to $\mathrm{V} / S$ ratio.

Indeed, the insertion of the six hemispheres impacts the volume and the surface of the cavity. If $V_{0}, S_{0}$ stand for the volume and the surface of the conventional RC, and $V_{1}, S_{l}$ stand for the same quantities in the chaotic RC respectively, then, the quality factors $Q_{0}$ and $Q_{1}$ in these two cavities verify $Q_{0} / \mathrm{Q}_{1}=\mathrm{V}_{0} \cdot \mathrm{S}_{1} /\left(\mathrm{V}_{1} \cdot \mathrm{S}_{0}\right)=1.12$.

Besides, the average ratio over the measure frequency band of the quality factors extracted from measurements in both cavities is of 1.15 . The agreement of this value with the theoretical prediction confirms the fact that the quality factor variation is due to the cavity geometries.

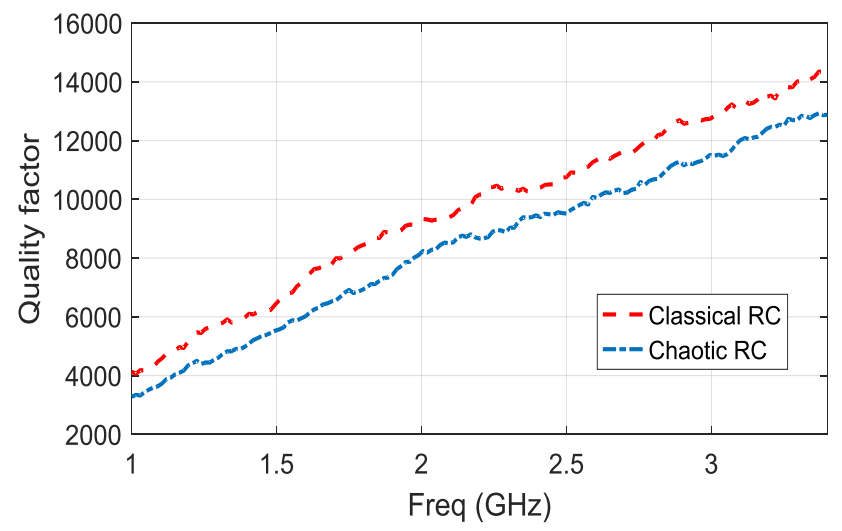

Fig. 7. Quality factors of the classical and the chaotic reverberation chambers.

\section{B. Antenna efficiency}

In this section, we present calculation results of the enhanced backscatter coefficient (Fig. 8) and both total (Fig. 9) and radiation (Fig. 10) antenna efficiencies. Results have been smoothed using a $100 \mathrm{MHz}$ sliding window in order to blur the frequency fluctuation of the parameters.

When considering the chamber's classical configuration, $e_{b}$ coefficient presents higher values in low frequencies. However, for frequencies above $1.5 \mathrm{GHz}$, and for both chamber configurations, the enhanced backscatter coefficient oscillates around two, which represents the theoretically ideal value of the backscatter coefficient. This result shows a good homogeneity of the electrical field inside the reverberation chamber and so a good stirring performance.

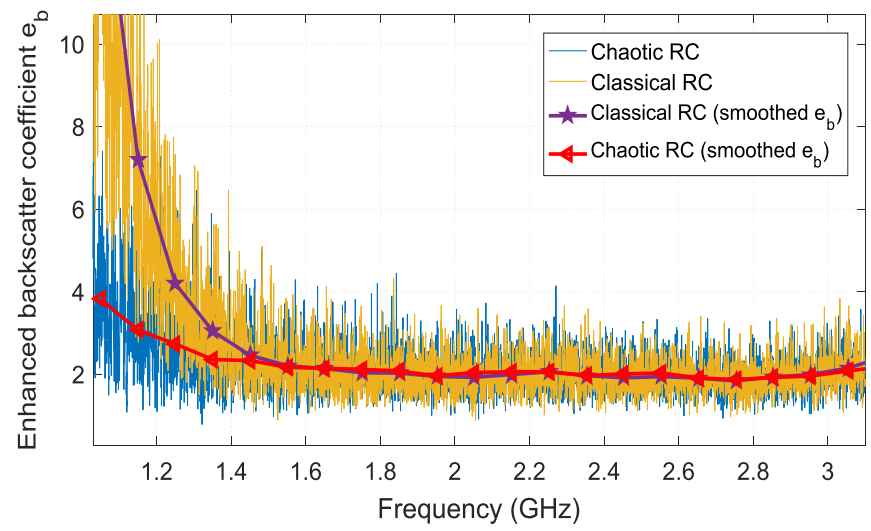

Fig. 8. Enhanced backscattering coefficient versus frequency in both RC configurations: classical and chaotic.

Once the enhanced backscattering coefficient as well as the quality factor are known, it is possible to compute the antenna total efficiency from (7). Results are presented in Fig. 9. We can see that both results (classical RC and chaotic RC) obtained when considering mechanical stirring associated to frequency smoothing are very close (with a maximal difference 
of $4 \%$ ). Indeed, as we work at a very high frequency compared to the RC LUF, even the classical cavity behaves close to an ideal RC. Also, we note that the only mechanical stirring is not sufficient to retrieve a smooth efficiency and the fluctuations in this case reach up to $30 \%$ of the efficiency.

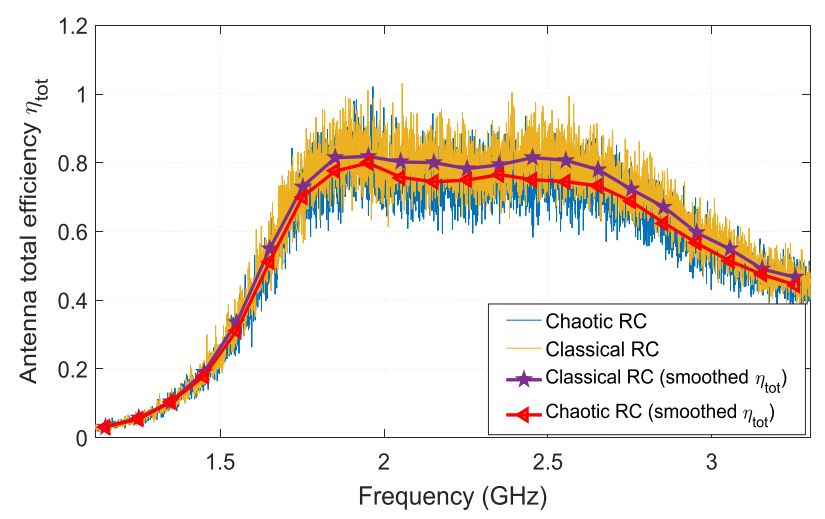

Fig. 9. Total efficiency of the patch antenna.

The antenna radiation efficiency can then be obtained from the total one by taking into account mismatches. Results are presented in Fig. 10. As the patch antenna is well matched in the $1.7-2.7 \mathrm{GHz}$ frequency band, the radiation efficiency is very close to the total one.

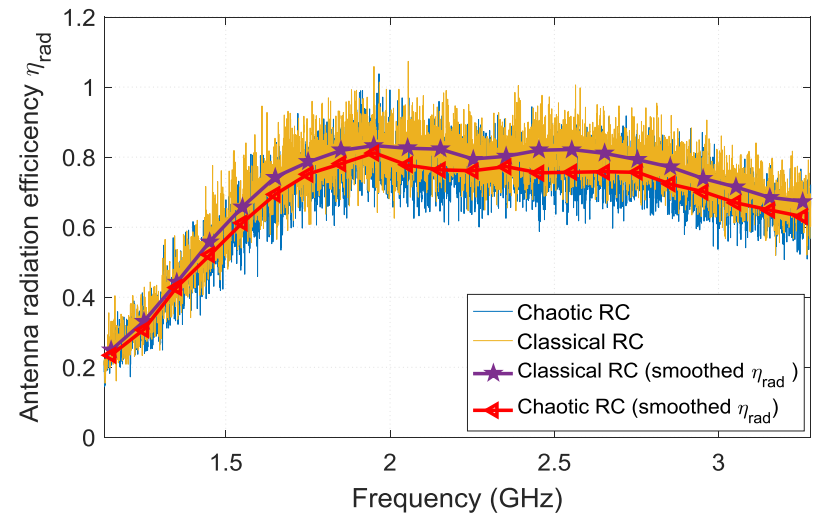

Fig. 10. Radiation efficiency of the patch antenna.

\section{CONCLUSION}

The radiation and total efficiencies of a slotted-based patch antenna have been evaluated within two configurations of a unique RC: the classical configuration and the chaotic one. Both mechanical and frequency stirring processes have been considered to average the measurement over several uncorrelated data. The performance study of these two stirring approaches shows that the chaotic cavity allows obtaining lower fluctuations of the reflection coefficient around its average value compared to the classical cavity. This enhancement of the RC properties thanks to the chaotic behavior is also confirmed by the enhancement backscattering coefficient in the lowest frequency range but the impact of the cavity configuration is not highlighted in the computed efficiency. Indeed, the considered frequency range is much higher than the RC LUF and thus, the RC is close to an ideal behavior, even in its classical configuration. Further work will include antenna efficiency measurement in a frequency range close to the LUF where the impact of the cavity chaoticity is expected to be enhanced.

\section{REFERENCES}

[1] M. Piette, "Antenna radiation efficiency measurements in a reverberation chamber," in Proc. Asia-Pacific Radio Science Conf., Aug. 24-27, pp. 19-22, 2004

[2] Christopher L.Holloway, Haider A.Shah, Ryan J.Pirkl, William F.Young, David A.Hill and John Ladbury, "Reverberation chamber techniques for determining the radiation and total efficiency of antennas". IEEE transactions on antennas and propagation, Vol .60, No 4, April, 2012.

[3] P. Besnier, J. Sol, A. Presse, C. Lemoine and A. C. Tarot, "Antenna efficiency measurement from quality factor estimation in reverberation chamber," European Microwave Conference (EuMC), London, pp. 715 718, 2016.

[4] Kent Madsén, Paul HallbjÖrner, and Charlie Orlenius, "Models for the number of independent samples in reverberation chamber measurements with mechanical, frequency, and combined stirring". IEEE antennas and wireless propagation letters, Vol. 3, 2004

[5] Christophe Lemoine, Philippe Besnier, M'Hamed Drissi, "Evaluation of frequency and mechanical stirring efficiency in a reverberation chamber", EMC Europe 2008, Sep 2008, Hambourg, Germany.

[6] J.-B. Gros, U. Kuhl, O. Legrand, F. Mortessagne, O.Picon, E.Richalot, "Statistics of the electromagnetic response of a chaotic reverberation chamber", Advanced Electromagnetics, Vol 4, No 2 (2015), pages 38-43.

[7] Zhihao Tian, Yi Huang and Qian Xu, "Enhanced backscatter coefficient measurement at high frequencies in reverberation chamber", International Workshop on Electromagnetics", Applications and Student Innovation Competition, London, UK, 2017. 